\title{
Barriers in Systemic Delivery and Preclinical Testing of Synthetic microRNAs in Animal Models: an Experimental Study on miR-215-5p Mimic
}

\author{
Tana MACHACKOVA ${ }^{1}$, Petra VYCHYTILOVA-FALTEJSKOVA ${ }^{1}$, Kamila SOUCKOVA ${ }^{1}$, \\ Richard LAGA ${ }^{2}$, Ladislav ANDROVIČ ${ }^{2}$, Gabriela MIXOVÁ ${ }^{2}$, Ondrej SLABY ${ }^{1,3}$ \\ ${ }^{1}$ Central European Institute of Technology, Masaryk University, Brno, Czech Republic, ${ }^{2}$ Institute of \\ Macromolecular Chemistry of the Czech Academy of Sciences, Prague, Czech Republic, \\ ${ }^{3}$ Department of Biology, Faculty of Medicine, Masaryk University, Brno, Czech Republic
}

Received September 3, 2020

Accepted March 11, 2021

Epub Ahead of Print May 12, 2021

\section{Summary}

Mus musculus is the most commonly used animal model in microRNA research; however, little is known about the endogenous miRNome of the animals used in the miRNAtargeting preclinical studies with the human xenografts. In the presented study, we evaluated the NOD/SCID gamma mouse model for the preclinical study of systemic miR-215-5p substitution with a semitelechelic poly[ $N$-(2-hydroxypropyl)methacrylamide]-based carrier conjugated with miR-215-5pmimic via a reductively degradable disulfide bond. Murine mmumiR-215-5p and human hsa-miR-215-5p have a high homology of mature sequences with only one nucleotide substitution. Due to the high homology of hsa-miR-215-5p and mmu-hsa-miR-215$5 p$, a similar expression in human and NOD/SCID gamma mice was expected. Expression of mmu-miR-215 in murine organs did not indicate tissue-specific expression and was highly expressed in all examined tissues. All animals included in the study showed a significantly higher concentration of miR-215-5p in the blood plasma compared to human blood plasma, where miR-215-5p is on the verge of a reliable detection limit. However, circulating mmu-miR-215-5p did not enter the human xenograft tumors generated with colorectal cancer cell lines since the levels of miR-215-5p in control tumors remained notably lower compared to those originally transfected with miR-215-5p. Finally, the systemic administration of polymer-miR-215-5p-mimic conjugate to the tail vein did not increase miR-215-5p in NOD/SCID gamma mouse blood plasma, organs, and subcutaneous tumors. It was impossible to distinguish hsa-miR-215-5p and mmu-miR-215-5p in the murine blood and organs due to the high expression of endogenous mmu-miR-215-5p. In conclusion, the examination of endogenous tissue and circulating miRNome of an experimental animal model of choice might be necessary for future miRNA studies focused on the systemic delivery of miRNA-based drugs conducted in the animal models.

\section{Key words}

miRNA • miR-215-5p • Animal model • Systemic delivery

\section{Corresponding author}

O. Slaby, Central European Institute of Technology (CEITEC), Masaryk University, Kamenice 5 (A35), 625 00, Brno, Czech Republic. E-mail: on.slaby@gmail.com

MicroRNAs (miRNAs) are highly conserved, small non-coding RNAs, 18-25 nucleotides in length. They act as post-transcriptional regulators of gene expression by either the post-transcriptional suppression of mRNA translation or induction of mRNA degradation (Lai 2002). MiRNAs expression is frequently either down-regulated or up-regulated in tumor tissue compared to healthy tissue (Ali Syeda et al. 2020). In the past decade, miRNAs were studied as potential biomarkers, therapeutic targets, or experimental drugs in various diseases, including cancer (Hanna et al. 2019). The use of animal models in cancer research significantly contributed to discoveries and rapid advances in 
biomedical technologies. Mus musculus is a widely used animal model in miRNA studies; however, little is known about the role of miRNome of Mus musculus in miRNAtargeting preclinical studies. Only a handful of studies examined miRNA profiles of model organisms, including Mus musculus (Takada et al. 2006, Pal and Kasinski 2017). Approximately $60 \%$ of mouse miRNA loci are conserved between mice and humans (Griffiths-Jones et al. 2008, Lagos-Quintana 2003). Despite the high degree of conservation of miRNAs across the species, remaining unconserved miRNAs can lead to significant obstacles in the studies focused on systemic delivery of miRNAs conducted in animal models. In the presented study, we evaluated NOD/SCID gamma mouse (NSG) as a model for the preclinical study of miR-215-5p substitution using a polymeric delivery system conjugated with miR-215-5p mimic.

MiR-215-5p is a small non-coding RNA acting as an oncogene or tumor suppressor in various malignant diseases. MiR-215-5p was found to have tumor suppressor effects in non-small cell lung cancer (Yao et al. 2018, Hou et al. 2015), breast cancer (Yao et al. 2017, Gao et al. 2019), mesothelioma (Singh et al. 2019), hepatocellular carcinoma (Ren et al. 2017), and thyroid cancer (Han et al. 2019). MiR-215-5p has been confirmed to serve as a tumor suppressor in colorectal cancer (CRC) (Vychytilova-Faltejskova et al. 2017). Interestingly, the oncogenic effects of miR-215-5p were observed in high-grade glioma (Wei et al. 2017) and gastric cancer (Chen et al. 2017, Li et al. 2016). MiR-215-5p was identified as a potential biomarker in several cancers, including CRC (Karaayvaz et al. 2011). However, the fact that miR-215-5p could serve as a potential therapeutic target or drug is far more interesting. Substitution of miR-215-5p in the CRC tumors might lead to a decrease of invasivity, metastatic potential, and inhibition of progression of the disease. However, the invention of the system for the targeted delivery of miR-215-5p and the appropriate animal model is necessary to uncover the full therapeutic potential of miR-215-5p in CRC. Unfortunately, mmumiR-215-5p and human hsa-miR-215-5p have a high homology of mature sequences with only one nucleotide substitution (Fig. 1A), which exclude NSG mouse from the list of suitable animal models for such study.

Currently, there are no commercial miRNA delivery systems available, except liposomal formulations, which cannot ensure the targeted delivery of the cargo to the tumor. Our study used a semitelechelic linear polymer based on poly[N-(2hydroxypropyl)methacrylamide] (PHPMA) with a terminal pyridyl disulfide group conjugated with a thiol group-terminated miR-215-5p mimic via disulfide bond formation (p(HPMA)n-S-S-miR-215-5p-mimic, Fig. 1B). Such a designed delivery system should provide miR215-5p with higher stability in the blood, prolong its circulation half-life and increase its accumulation in the tumor cells due to enhanced permeability and retention effect (Golombek et al. 2018). The disulfide bond between the polymer chain and the miRNA should be cleaved due to the increased concentration of glutathione inside the cells, leading to the intracellular release of free miRNA. The sequence and chemical modification of miR-215-5p mimic was 5'-rArUrG rArCrC rUrArU rGrArA rUrUrG rArCrA rGrArC-3' and 5'-rUrCrU rGrUrC rArUrU rUrCrU rUrUrA rGrGrC rCrArA rUrA/3ThioMC3-D/-3" (" $r$ " in the sequence means RNA).

In vivo studies were performed in NSG mice (obtained from The Jackson Laboratory, Bar Harbor, ME, USA) housed and monitored in an individually ventilated cage system (Techniplast, Buguggiate, Italy) with ad libitum access to water and feeding. Following national and EU animal welfare legislation, animal experiments were performed, and all procedures were approved by institutional (Masaryk University, Brno, Czech Republic) and national ethics committees. Identification number of project MSMT-9643/2017-3. Xenograft subcutaneous tumors were generated in thirtyfive NSG mice in total using HCT-116, RKO, and HCT15 cells. Mice were anesthetized by intraperitoneal injection of xylazine $(100 \mathrm{mg} / \mathrm{kg}$ of weight $) / \mathrm{ketamine}$ $(10 \mathrm{mg} / \mathrm{kg}$ of weight). The injected volume of cell suspension was $2.5 \times 10^{6}$ cells per $100 \mu \mathrm{l}$ of PBS. Tumor growth and animal behavior were individually monitored during the experiment. For evaluation of miR-215-5p expression pre- and post-implantation, in total, eighteen mice were used for subcutaneous injection of HCT-116 (6 animals), RKO (6 animals), and HCT-15 cells (6 animals). Mice were sacrificed, and an autopsy was performed according to palpation tests - after 29 days (HCT-116), after 22 days (RKO), and after 15 days (HCT-15). Subcutaneous tumors were collected and stored at $-80^{\circ} \mathrm{C}$. Additionally, to examine miR-215-5p expression in organs, blood plasma and organs were collected from fifteen NSG mice. Moreover, two NU/NU mice and an individual of Rat ratticus were included in the study, and their organs and blood plasma were 
collected to examine miR-215-5p expression level in other rodent species. Lastly, pre-operative blood plasma samples of 8 patients planned for microdiscectomy for disc herniation in the Faculty Hospital Brno (Brno, Czech Republic) were used. The patients signed an informed consent form. To evaluate the distribution of LS4 + miR$215-5 \mathrm{p}$, the remaining seventeen NSG mice with HCT116 subcutaneous tumors were used. One animal was excluded from the study due to small tumor size. After fourteen days, $217 \mu \mathrm{l}$ of suspension of the LS4 + miR$215-5 \mathrm{p} \quad(7 \mathrm{mg} / \mathrm{kg}, \quad 5 \mathrm{mg} / \mathrm{kg}, \quad 2.5 \mathrm{mg} / \mathrm{kg}, \quad 1 \mathrm{mg} / \mathrm{kg})$ conjugate was administered via tail vein to three animals in each group. As a control $217 \mu \mathrm{l}$ Tris-EDTA (TE) buffer was administered to two animals, and $217 \mu \mathrm{l}$ of suspension of LS4 (6.54 g/l) in TE buffer was administered to two animals. Animals were sacrificed after $48 \mathrm{~h}$. Organs and blood plasma were collected, processed, and stored at $-80{ }^{\circ} \mathrm{C}$. Collection of blood plasma was successful only in one animal in group LS4 + TE buffer. Total RNA enriched with a fraction of small RNAs from mice and human blood plasma samples were isolated using miRNeasy Serum/Plasma Kit (Qiagen, USA). Quantity and quality of RNA were measured using NanoDrop 2000 Spectrophotometer (Thermo Fisher Scientific, USA). Total RNA enriched with a fraction of small RNAs was isolated using mirVana miRNA Isolation Kit (Ambion, Austin, TX, USA) in the case of subcutaneous tumors and organs and tissues from mice. Direct-zol RNA Microprep Kit (Zymo Research, Irvine, CA, USA) was used to isolate RNA from cell lines according to the manufacturer's instructions. Quality and quantity of isolated RNA were measured spectrophotometrically using NanoDrop 2000c (Thermo Fisher Scientific, Waltham, MA, USA) and fluorometrically using Qubit 2.0 and Qubit BR RNA Assay Kit (Thermo Fisher Scientific). Isolated RNA was stored at $-80{ }^{\circ} \mathrm{C}$ until further processing. Expression levels of miR-215-5p were measured by a quantitative real-time polymerase chain reaction (qRT-PCR). MiRNA reverse transcription was performed using $6.67 \mathrm{ng}$ of total RNA, gene-specific primers (hsa-miR-215-5p; ID 000518, RNU48; ID 001006 (Applied Biosystems, Foster City, CA, USA)), and TaqMan ${ }^{\mathrm{TM}}$ MicroRNA Reverse Transcription Kit according to the TaqMan MicroRNA Assay protocol (Applied Biosystems, Foster City, CA, USA). MiRNA qRT-PCR was performed using TaqMan $^{\mathrm{TM}}$ Universal Master Mix II, no UNG (Applied Biosystems, Foster City, CA, USA) according to the manufacturer's recommendations. In xenograft tumors,
RNU48 was used as an endogenous control and miR-215$5 \mathrm{p}$ expression was normalized using formula $\mathrm{Ct}(\mathrm{miR}-$

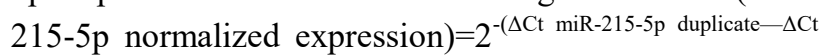
RNU48 duplicate). MiR-215-5p measured in blood plasma and in murine tissues was normalized using the Ct-40 normalization approach with formula $\mathrm{Ct}(\mathrm{miR}-215-5 \mathrm{p}$ normalized expression $)=2^{-(\Delta \mathrm{Ct}}$ miR-215-5p duplicate-40) since RNU48 is not suitable endogenous control for murine samples. An unpaired parametric two-sided $t$-test was used to analyze expression data. Analyses were done using GraphPad Prism 8 (GraphPad Software, San Diego, CA, USA). P-values of less than 0.05 were considered statistically significant.

Analyses of miR-215-5p expression levels in blood plasma showed surprising results. All rodents included in the study had a significantly higher concentration of miR-215-5p in the blood plasma compared to human blood plasma (Fig. 1C). In human blood plasma, miR-215-5p had relatively low expression in comparison to miR-215-5p expression in blood plasma of NSG mice $\log 2$ (fold change over the Ct-40)=9.32; $\mathrm{P}=0.0233$, in blood plasma of NU/NU mice $\log 2$ (fold change over the $\mathrm{Ct}-40)=9.16 ; \mathrm{P}<0.0001$, and $\log 2$ (fold change over the Ct-40) $=9.63$ in blood plasma of Rattus norvegicus. Despite the high sequence homology between hsa-miR-215-5p and mmu-miR-215-5p, functions of mmu-miR-215-5p and hsa-miR-215-5p will probably differ in both species. In our previous studies using CRC patients samples, we observed hsa-miR-215-5p to have high expression in colon epithelium and low expression in blood plasma. In the literature, miR-215-5p is usually reported in tissues like colon and small intestine, and its most notable role is the regulation of epithelial-mesenchymal transition (VychytilovaFaltejskova et al. 2017). In the accessible resources, there is a lack of information on miR-215-5p expression in the mouse. On the other hand, several studies are showing the expression of miR-215 in Rattus norvegicus. Specific expression of miR-215 was described in rat ischemic brain and heart; moreover, a high number of miR-215 isomiRs was detected in rat intestine (Jeyaseelan, Lim, and Armugam 2008, Smith et al. 2016, Vacchi-Suzzi et al. 2012). Analysis of miR-215-5p expression in rodent organs showed high expression of miR-215-5p in all collected organs, especially in the liver and kidneys (Fig. 1D). However, the expression of miR-215-5p in the liver and kidneys was highly variable. Expression analyses of xenograft tumors using CRC cells stable transfected with miR-215-5p or empty vector as a control 
showed an inability of mmu-miR-215-5p to enter the xenograft tumor. CRC cell lines used for subcutaneous injection had similar expression of miR-215-5p as tumors of their origin (Fig. 1E). These results indicate the validity of NSG mice for in vivo experiments with stable transfected cell lines and NSG mice can be considered a suitable animal model for the study of miRNA influence on tumor growth and metastatic potential.
A $\begin{array}{ll}\text { hsa-miR-215-5p } & \text { AUGACCUAUGAAUUGACAGAC } \\ \text { mmu-miR-215-5p } & \text { AUGACCUAUGAUUUGACAGAC }\end{array}$

B

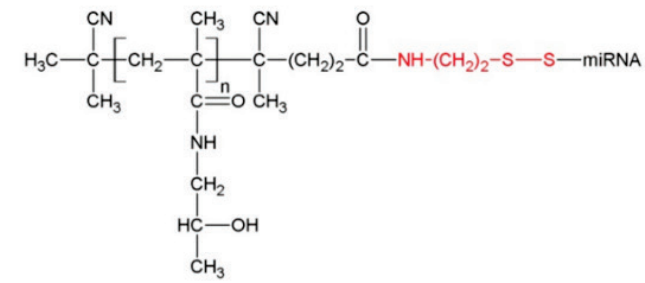

D

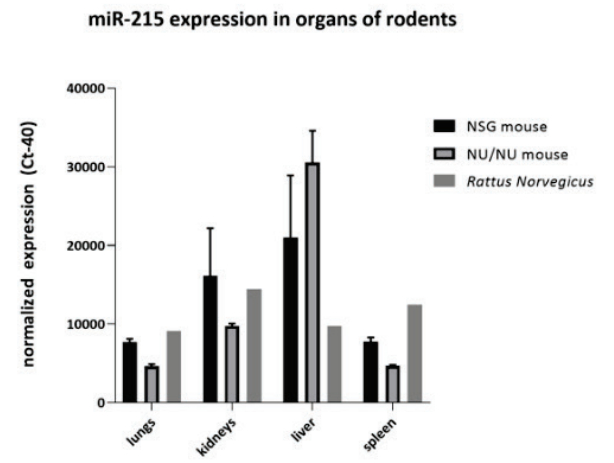

C

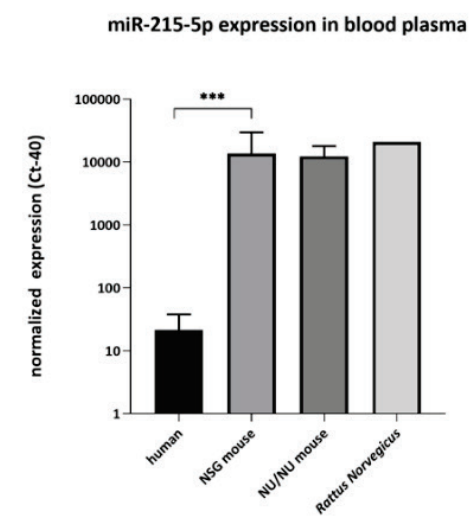

$\mathbf{E}$

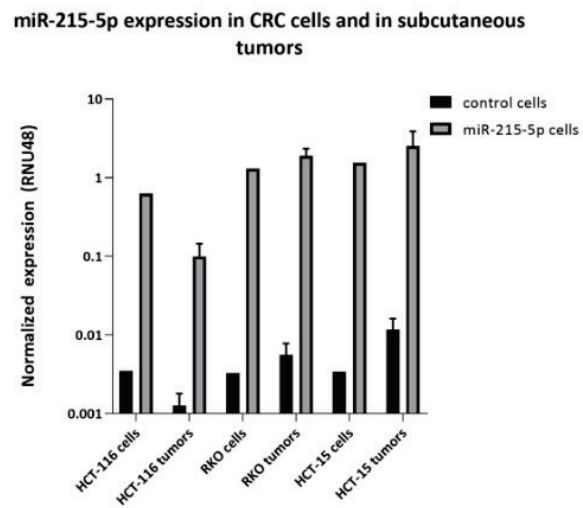

Fig. 1. (A) Sequence homology of mature hsa-miR-215-5p and mmu-miR-215-5p. (B) Semitelechelic linear homopolymer with a terminal pyridyl disulfide group conjugated with miR-215-5p mimic (miRNA-SH passenger strand). (C) Expression levels of miR-215-5p in the blood plasma of humans, NSG mice, NU/NU mice, and Rattus norvegicus. (D) Expression levels of miR-215-5p in organs of NSG mice, $\mathrm{NU} / \mathrm{NU}$ mice, and Rattus norvegicus. (E) Expression levels of miR-215-5p in implanted cell lines (control, miR-215-5p transfected) and related xenograft tumors in NSG mice (control, miR-215-5p transfected).

Expression analyses of miR-215-5p in blood plasma and tissue specimens after systemic administration of LS4 + miR-215 did not reveal any significant increase in miR-215-5p levels independently on the concentration (Fig. 2A, B). However, differences in expression were not probably detected due to high endogenous mmu-miR-215-5p expression levels. The dramatic difference in miR-215-5p expression between species and high level of sequence homology (one nucleotide substitution $U>A$ ) disabled the usage of species-specific miR-215-5p qPCR assays, and the hsa-miR-215-5p levels in murine blood plasma/tissue could not be accurately distinguished from mmu-miR215-5p. Unfortunately, high expression of miR-215-5p in murine blood plasma excludes Mus musculus as a suitable animal model for studying the systemic administration of miR-215-5p based drugs.

Considering these findings, it is highly probable that many more human miRNAs have significantly different function and expression levels in animal models and humans. However, little research was dedicated to comparing normal human and animal models' miRNA profiles. Thus many miRNAs candidates in the preclinical animal studies might interfere with the endogenous miRNome of the animal. This issue might require use of transgenic animal models or fluorescent labeling of carrier-miRNA conjugates. In conclusion, the examination of endogenous tissue and circulating miRNome of an experimental animal model of choice might be necessary for future miRNA studies focused on the systemic delivery of miRNA based drugs conducted in animal models. 
A

miR-215-5p expression in blood plasma

- systemic administration of LS4 + miR-215

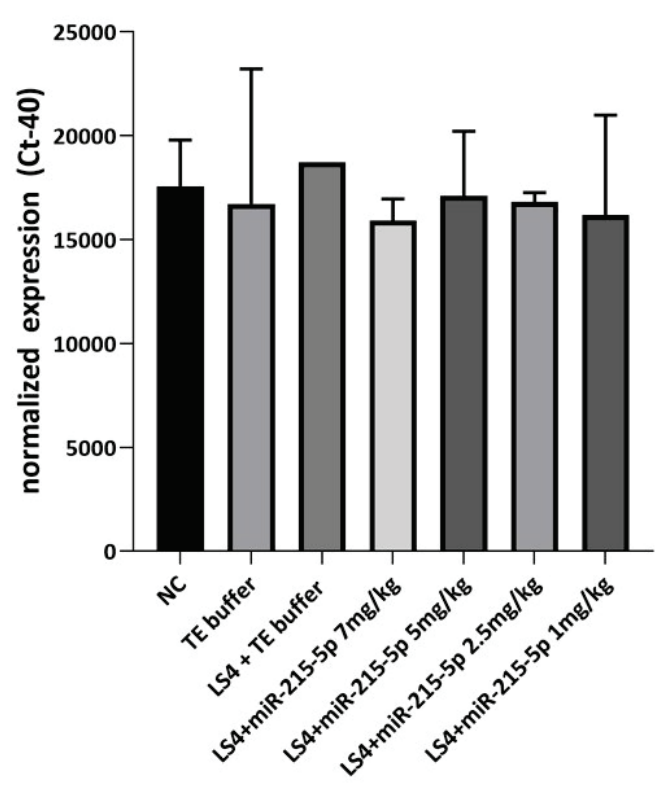

Fig. 2. (A) Expression levels of miR-215-5p in NSG mice blood plasma after systemic dministration of LS4 + miR-215-5p conjugate. (B) Expression levels of miR-215-5p in NSG mice organs after systemic administration of LS4 + miR-215-5p conjugate.

B

miR-215-5p expression in organs and subcutaneous tumors

- systemic administration of LS4 + miR-215

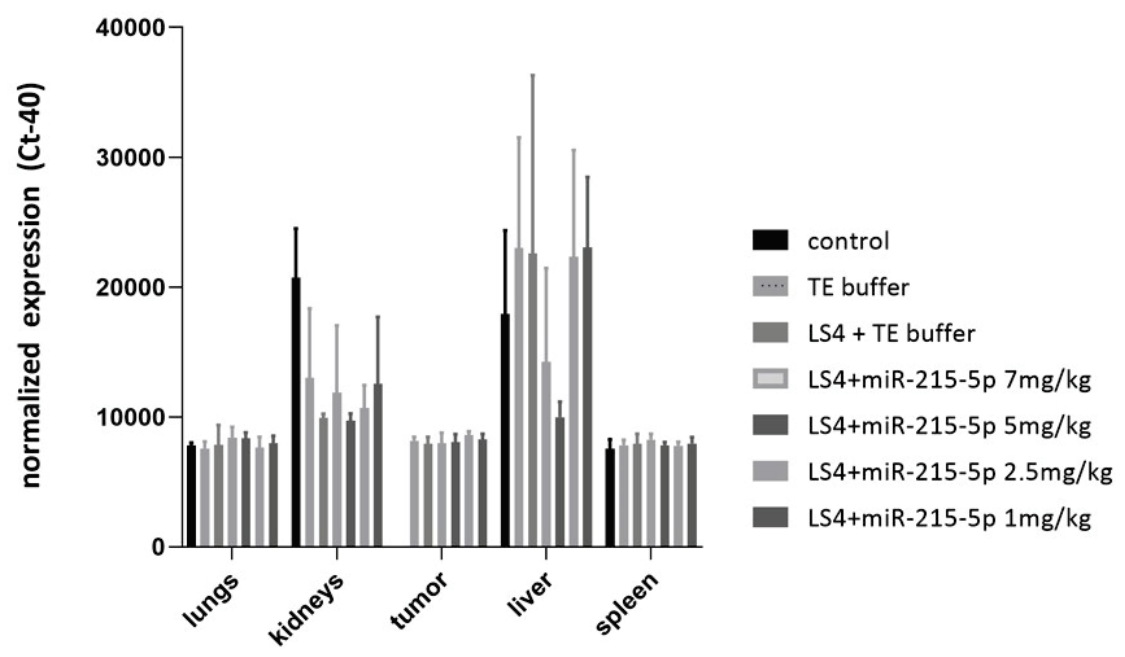

\section{Conflict of Interest}

There is no conflict of interest.

\section{Acknowledgements}

This work was supported by grant project GACR 16-18257S.

\section{Abbreviations}

CRC - colorectal cancer, miRNA - microRNA, NSG NOD/SCID gamma, PHPMA - poly[N-(2hydroxypropyl)methacrylamide], qRT-PCR quantitative real-time polymerase chain reaction, TE buffer - Tris-EDTA buffer.

\section{References}

ALI SYEDA Z, SARATU' LANGDEN SS, MUNKHZUL C, LEE M, SONG SJ: Regulatory mechanism of MicroRNA expression in cancer. Int J Mol Sci 21: 1723, 2020. https://doi.org/10.3390/ijms21051723. 
CHEN Z, LIU K, LI L, CHEN Y, SHOUQIN D: mir-215 promotes cell migration and invasion of gastric cancer by targeting retinoblastoma tumor suppressor gene 1. Pathol Res Pract 213: 889-894, 2017. https://doi.org/10.1016/i.prp.2017.06.006

GAO JB, ZHU MN, ZHU XL: miRNA-215-5p suppresses the aggressiveness of breast cancer cells by targeting Sox9. FEBS Open Bio 9: 1957-1967, 2019. https://doi.org/10.1002/2211-5463.12733

GOLOMBEK SK, MAY J-N, THEEK B, APPOLD L, DRUDE N, KIESSLING F, LAMMERS T: Tumor targeting via EPR: Strategies to enhance patient responses. Adv Drug Deliv Revs 130: 17-38, 2018. https://doi.org/10.1016/j.addr.2018.07.007

GRIFFITHS-JONES S, SAINI HK, VAN DONGEN S, ENRIGHT AJ: miRBase: tools for microRNA genomics. Nucleic Acids Res 36 (Database issue): D154-D158, 2008. https://doi.org/10.1093/nar/gkm952

HAN J, ZHANG M, NIE C, JIA J, WANG F, YU J, BI W, LIU B, SHENG R, HE G, KONG L, ZHENG K, PANG R, DING Z, CEN L, ET AL:: mir-215 suppresses papillary thyroid cancer proliferation, migration, and invasion through the AKT/GSK-3ß/Snail signaling by targeting ARFGEF1. Cell Death Dis 10: 195, 2019. https://doi.org/10.1038/s41419-019-1444-1

HANNA J, HOSSAIN GS, KOCERHA J: The potential for microRNA therapeutics and clinical research. Front Genet 10: 478, 2019. https://doi.org/10.3389/fgene.2019.00478

HOU Y, ZHEN J, XU X, ZHEN K, ZHU B, PAN R, ZHAO C: miR-215 functions as a tumor suppressor and directly targets ZEB2 in human non-small cell lung cancer. Oncol Lett 10: 1985-1992, 2015. https://doi.org/10.3892/ol.2015.3587

JEYASEELAN K, LIM KY, ARMUGAM A: MicroRNA expression in the blood and brain of rats subjected to transient focal ischemia by middle cerebral artery occlusion. Stroke 39: 959-966, 2008. https://doi.org/10.1161/STROKEAHA.107.500736

KARAAYVAZ M, PAL T, SONG B, ZHANG C, GEORGAKOPOULOS P, MEHMOOD S, BURKE S, SHROYER K, JU J: Prognostic significance of miR-215 in colon cancer. Clin Colorectal Cancer 10: 340-347, 2011. https://doi.org/10.1016/j.clcc.2011.06.002

LAGOS-QUINTANA M, RAUHUT R, MEYER J, BORKHARDT A, TUSCHL T: New microRNAs from mouse and human. RNA 9: 175-179, 2003. https://doi.org/10.1261/rna.2146903

LAI EC: Micro RNAs are complementary to 3' UTR sequence motifs that mediate negative post-transcriptional regulation. Nat Genet 30: 363-364, 2002. https://doi.org/10.1038/ng865

LI N, ZHANG Q-Y, ZOU JL, LI Z-W, TIAN T-T, DONG B, LIU X-J, GE S, ZHU Y, GAO J, SHEN L: MiR-215 promotes malignant progression of gastric cancer by targeting RUNX1. Oncotarget 7: 4817-4828, 2016. https://doi.org/10.18632/oncotarget.6736

PAL AS, KASINSKI AL: Animal models to study microRNA function. Adv Cancer Res 135: 53-118, 2017. https://doi.org/10.1016/bs.acr.2017.06.006

REN Y, SHANG J, LI J, LIU W, ZHANG Z, YUAN J, YANG M: The long noncoding RNA PCAT-1 links the microRNA MiR-215 to oncogene CRKL-mediated signaling in hepatocellular carcinoma. J Biol Chem 292: 17939-17949, 2017. https://doi.org/10.1074/jbc.M116.773978

SINGH A, BHATTACHARYYA N, SRIVASTAVA A, PRUETT N, TAYLOR RIPLEY R, SCHRUMP DS, HOANG CD: MicroRNA-215-5p treatment suppresses mesothelioma progression via the MDM2-P53-signaling axis. Mol Ther 27: 1665-1680, 2019. https://doi.org/10.1016/j.ymthe.2019.05.020

SMITH A, CALLEY J, MATHUR S, QIAN H-R, WU H, FARMEN M, CAIMENT F, BUSHEL PR, LI J, FISHER C, KIRBY P, KOENIG E, HALL DG, WATSON DE: The rat microRNA body atlas; Evaluation of the microRNA content of rat organs through deep sequencing and characterization of pancreas enriched miRNAs as biomarkers of pancreatic toxicity in the rat and dog. BMC Genomics 17: 694, 2016. https://doi.org/10.1186/s12864-016-2956-Z

TAKADA S, BEREZIKOV E, YAMASHITA Y, LAGOS-QUINTANA M, KLOOSTERMAN WP, ENOMOTO M, HATANAKA H, FUJIWARA S, WATANABE H, SODA M, CHOI YL, PLASTERK RHA, CUPPEN E, MANO H: Mouse microRNA profiles determined with a new and sensitive cloning method. Nucleic Acids Res 34: e1 15, 2006. https://doi.org/10.1093/nar/gk1653 
VACCHI-SUZZI C, BAUER Y, BERRIDGE BR, BONGIOVANNI S, GERRISH K, HAMADEH HK, LETZKUS M, LYON J, MOGGS J, PAULES RS, POGNAN F, STAEDTLER F, VIDGEON-HART MP, GRENET O, COUTTET P: Perturbation of microRNAs in rat heart during chronic doxorubicin treatment. PLoS One 7: e40395, 2012. https://doi.org/10.1371/journal.pone.0040395

VYCHYTILOVA-FALTEJSKOVA P, MERHAUTOVA J, MACHACKOVA T, GUTIERREZ-GARCIA I, GARCIASOLANO J, RADOVA L, BRCHNELOVA D, SLABA K, SVOBODA M, HALAMKOVA J, DEMLOVA R, KISS I, VYZULA R, CONESA-ZAMORA P, SLABY O: MiR-215-5p is a tumor suppressor in colorectal cancer targeting EGFR ligand epiregulin and its transcriptional inducer HOXB9. Oncogenesis 6: 399, 2017. https://doi.org/10.1038/s41389-017-0006-6

WEI Y, SUN J, LI X: MicroRNA-215 enhances invasion and migration by targeting retinoblastoma tumor suppressor gene 1 in high-grade glioma. Biotechnol Lett 39: 197-205, 2017. https://doi.org/10.1007/s10529-016-2251-8

YAO J, ZHANG P, LI J, XU W: MicroRNA-215 acts as a tumor suppressor in breast cancer by targeting AKT serine/threonine kinase 1. Oncol Lett 14: 1097-1104, 2017. https://doi.org/10.3892/ol.2017.6200

YAO Y, SHEN H, ZHOU Y, YANG Z, HU T: MicroRNA-215 suppresses the proliferation, migration and invasion of non-small cell lung carcinoma cells through the downregulation of matrix metalloproteinase-16 expression. Exp Ther Med 15: 3239-3246, 2018. https://doi.org/10.3892/etm.2018.5869 